\title{
Diversidad del género Polylepis (Rosaceae, Sanguisorbeae) en los Andes peruanos
}

\author{
Diversity of the genus Polylepis (Rosaceae, Sanguisorbeae) in the Peruvian \\ Andes
}

Wilfredo Mendoza ${ }^{1}$ y Asunción Cano ${ }^{1,2}$

1 Laboratorio de florística, Departamento de Dicotiledóneas, Museo de Historia Natural, Universidad Nacional Mayor de San Marcos (UNMSM) Av. Ar San Marcos 11 , Per Email Wilfredo Mendoza: wilfredomen@gmail.com

2 Instituto de Investigación de Ciencias Biológicas Antonio Raimondi (ICBAR), Facultad de Ciencias Biológicas, Universidad Nacional Mayor de San Marcos.

$\begin{array}{ll}\text { Presentado: } & 22 / 02 / 2011 \\ \text { Aceptado: } & 07 / 07 / 2010\end{array}$ Publicado online: $25 / 08 / 2011$

\begin{abstract}
Resumen
El presente estudio brinda información sobre la diversidad de las especies peruanas de Polylepis. Se reporta 19 especies (más del $70 \%$ de las 27 registradas para toda el área Andina), convirtiéndose el Perú en el país con mayor diversidad de Polylepis, en comparación a Bolivia (13), Ecuador (7), Argentina (4), Colombia (3), Chile (2) y Venezuela (1). Estas especies se distribuyen en 19 departamentos, encontrándose la mayor cantidad en Cusco (10) y Ayacucho (8). La mayor diversidad de especies (15) se registró en los Andes del sur, considerándose ésta región como el probable centro de diversificación del género Polylepis. Con respecto a la distribución altitudinal, la mayor diversidad de especies (18) se encuentra entre los 3000 y 4000 m de altitud.

Palabras claves: Polylepis, diversidad, endémicas, distribución, Perú.
\end{abstract}

\section{Abstract}

This study provides information on the diversity of Peruvian species of Polylepis. Nineteen (19) species are reported here (more than $70 \%$ of the 27 species registered for the whole Andean region). As a result, Peru could be considered as the country with the largest diversity of Polylepis species, in comparison with Bolivia (13), Ecuador (7), Argentina (4), Colombia (3), Chile (2), and Venezuela (1). The species occur in 19 departments of Peru, with the majority of them in Cusco (10) and Ayacucho (8). Species diversity is mostly concentrated in the Peruvian southern Andes (15 species), with the region becoming the potential center of diversification of the genus Polylepis. Regarding their altitudinal distribution, the greatest diversity (18) is found between 3000 and $4000 \mathrm{~m}$.

Keywords: Polylepis, diversity, endemics, distribution, Peru.

\section{Introducción}

La familia Rosaceae comprende alrededor de 100 géneros y 3000 especies, con una distribución casi cosmopolita; es dividida en cuatro subfamilias y 15 tribus (Romoleroux 1992, 1996). La tribu Sanguisorbeae presenta 14 géneros de gran interés biogeográfico, porque están distribuidos casi en todos los continentes, la mayor diversidad y centro principal de diversificación, se encuentra en el hemisferio sur, donde los géneros Polylepis, Tetraglochin, Margyricarpus y Acaena representan a Sudamérica (Pérez De Paz 2004).

En los bosques montanos y altoandinos de la Cordillera de los Andes, desde Venezuela hasta el norte de Chile y Argentina, con una población extratropical en el noreste y centro de Argentina, se distribuye el género Polylepis; que incluye alrededor de 27 especies (Simpson 1979, Cabido \& Acosta 1985, Kessler 1995, Romoleroux 1996, Mendoza 2005, 2010). Siendo principalmente árboles o arbustos que crecen sobre el límite superior de los bosques.

Existe gran interés ecológico, sistemático y biogeográfico por el género Polylepis, porque representa un sistema biológico único en los Andes, caracterizado por tener distribución restringida (Koepcke 1961, Servat et al. 2002).

La riqueza específica del género Polylepis para el Perú, fue difícil de precisar. Algunos autores estimaban entre 10 a 14 especies. Así para Bitter (1911), Macbride (1983) y Herrera (1943) reportan 12 especies, Simpson (1979) y Brako \& Zarucchi (1993) 10 especies, Fjelsa \& Kessler (1996) 11 especies, y Kessler \& Schmidt-Lebuhn (2006) 14 especies.

Mendoza (2010) en la más reciente revisión para el Perú, estableció en 19 especies la riqueza específica del género Polylepis, el presente reporte es parte de este estudio y brindar información sobre la distribución de la diversidad específica del género Polylepis para el Perú.

\section{Área de estudio}

El estudio se desarrollo en la Cordillera de los Andes del Perú, sobre los $1800 \mathrm{~m}$ de altitud, en el flanco oriental, hasta los 5100 m de altitud; abarcando principalmente la parte alta, tanto de la Cordillera Occidental, Oriental y de los valles interandinos de todo el territorio peruano, donde se encuentran establecidos los bosques de Polylepis. El estudio comprendió los departamentos(ordenados de norte a sur) de: Piura, Lambayeque, Cajamarca, Amazonas, La Libertad, San Martín, Ancash, Huánuco, Lima, Pasco, Junín, Huancavelica, Ayacucho, Apurímac, Cusco, Arequipa, Puno, Moquegua y Tacna.

\section{Material y métodos}

La información que se presenta es el producto de datos obtenidos por los autores desde hace más de 10 años (a partir de 1997), como resultado del estudio del género Polylepis para el Perú; alcanzándose a estudiar aproximadamente 429 ejemplares de Polylepis, ya sea por trabajo de campo o revisión de material de herbario.

Revisión de material de Herbario.- Se estudiaron todos los ejemplares de Polylepis, que se encuentran depositados en: Herbario de la Universidad Nacional de Cajamarca (CPUN), Herbario Cesar Vargas (CUZ), Field Museum de Chicago (F), Herbarium Areqvipense (HUSA), Missouri Botanical Garden (MO), US National Arboretum Washington (NA) y Herbario San Marcos (USM).

Trabajo de Campo.- Se exploró los Andes de Perú, desde 1997, como parte del "Proyecto Queñual" (dirigido por Grace Servat), que estuvo orientado a la investigación, educación, 
Tabla 1. Riqueza de especies del género Polylepis en los países de distribución y endemismo.

\begin{tabular}{lcc}
\hline País & Especies & Endemismo \\
\hline Perú & 19 & 5 \\
Bolivia & 13 & 4 \\
Ecuador & 7 & 2 \\
Argentina & 4 & 1 \\
Colombia & 3 & 1 \\
Chile & 2 & 0 \\
Venezuela & 1 & 0 \\
\hline
\end{tabular}

conservación y manejo de los bosques de Polylepis en el Perú. Este proyecto permitió realizar los estudios de campo de los bosque de Polylepis que se encuentran en los departamentos de Tacna, Puno, Arequipa, Cusco, Lima y Ancash. Posteriores exploraciones a otros departamentos donde se distribuye Polylepis, se realizaron con el apoyo de los proyectos desarrollados en el Laboratorio de Florística del Museo de Historia Natural (UNMSM).

\section{Resultados}

Diversidad.- Se registraron un total de 19 especies del género Polylepis para los Andes peruanos, que representa más del $70 \%$ de las 27 especies registradas en todos los Andes, superando a Bolivia que tiene el 40\% y Ecuador el 25\%. En los restantes países andinos, donde se distribuye este género está representado solamente de $14 \%$ a 3\% (Tabla 1 ).

El centro de diversificación del género Polylepis serian los Andes del sur del Perú, donde se registran 15 especies. Mientras que podemos considerar los Andes del norte de Perú, como el centro de origen del género Polylepis, debido a que en esta región se han registrado a dos especies más primitivas, $P$. multijuga Pilger y $P$. pauta Hieron., la primera restringida solamente a este sector de los Andes.

Endemismo.- De todas las especies encontradas en el Perú, cinco se distribuyen solamente en los Andes peruanos: Polylepis canoi W. Mendoza, P. flavipila (Bitter) M. Kessler \& SchmidtLeb., P. multijuga Pilger, P. racemosa Ruiz \& Pav. y P. subsericans J.F. Macbride, que representa el $26 \%$ del total de especies del género en el Perú.

Es importante indicar que cuatro especies no se encuentran en ninguna área protegida por el estado peruano: $P$. incarum (Bitter) M. Kessler \& Schmidt-Leb., P. lanata (Kuntze) M. Kessler \& Schmidt-Leb., P. subsericans J. F. Macbride y P. triacontandra Bitter.

Diversidad a nivel de departamentos.- El análisis de la riqueza departamental de las especies de Polylepis, indica que el departamento con mayor riqueza es Cusco con 10 especies, seguido por Ayacucho con 8. Le siguen Ancash, Junín y Lima con 6, Apurímac con 5 y Puno con 4. En contraste, los que tienen menos diversidad son los departamentos de Amazonas y Piura con una sola especie.

Polylepis se encuentra distribuido en 19 departamentos, de los 24 departamentos del Perú (Tabla 2). No se ha registrado ninguna especie en los departamentos de Tumbes e Ica de la costa peruana; y en Loreto, Ucayali y Madre de Dios de la Amazonía peruana. Los departamentos de Cusco y Ayacucho, son los más diversos, porque cuentan con una gran diversidad
Tabla 2. Riqueza de especies del género Polylepis, en los departamentos de Perú.

\begin{tabular}{lc}
\hline Departamentos & Número de especies registradas \\
\hline Cusco & 10 \\
Ayacucho & 8 \\
Ancash & 6 \\
Junín & 6 \\
Lima & 6 \\
Apurímac & 5 \\
Puno & 4 \\
Arequipa & 3 \\
Cajamarca & 3 \\
La Libertad & 3 \\
Tacna & 3 \\
Huánuco & 2 \\
Huancavelica & 2 \\
Lambayeque & 2 \\
Moquegua & 2 \\
Pasco & 2 \\
San Martín & 2 \\
Amazonas & 1 \\
Piura & 1 \\
\hline
\end{tabular}

de ecosistemas, que van desde las yungas (ceja de selva) en la vertiente oriental, valles interandinos, puna húmeda y seca. En cambio los departamentos, con menor número de especies, cuentan uno o dos de estos ecosistemas.

Distribución altitudinal.- El 94\% (18) de las especies que se encuentran en el Perú, están concentradas en el rango altitudinal de 3000 a $4000 \mathrm{~m}$, siendo Polylepis subsericans registrada a mayor altitud sobre los $5100 \mathrm{~m}$ (Mendoza 2005) para la Cordillera Vilcanota en Perú, y la especie registrada a menor altitud en Perú es $P$. pauta a tan solamente $1800 \mathrm{~m}$, en la Cordillera de Accanacu en el departamento de Cusco (Tabla 3).

\section{Discusión}

Las variaciones en los registros del número de especies de Polylepis para el Perú, se debió principalmente al limitado trabajo de campo que realizaron los investigadores, quienes exploraron áreas restringidas de los Andes peruanos y basaron sus estudios principalmente en la revisión de especímenes, que se hallaban en los herbarios; además muchas especies sufrieron cambios en su estatus en los diferentes tratamientos (Mendoza 2010).

También era muy frecuente denominar varías especies del Perú como una sola, así a $P$. weberbaueri, $P$. reticulata y $P$. microphylla se nombraba como $P$. weberbaueri; a $P$. flavipila y $P$. subsericans se identificaba como $P$. subsericans y a $P$. rugulosa, $P$. subtusalbida y $P$. lanata se denominaba como $P$. besseri, esta última especie no se encuentra en el Perú, su distribución está limitada para Bolivia (Mendoza et al. 2010).

Posiblemente todas las especies llegan a producir híbridos debido a que muchas veces ocurren dos hasta tres especies en la misma localidad (Simpson 1979, Mendoza 2005). La hibridización en el género Polylepis es considerada común, observándose poblaciones híbridas en Bolivia y Ecuador (Simpson 1979, 
Tabla 3. Lista de las especies de Polylepis reportadas para el Perú, con rangos altitudinales, distribución departamental (las abreviaturas usadas para los departamentos fueron tomados según Brako \& Zarucchi 1993).

\begin{tabular}{lll}
\hline Especies & Altitud (m) & \multicolumn{1}{c}{ Departamento } \\
\hline Polylepis canoi W. Mendoza & $3350-3400$ & AY, CU, JU \\
Polylepis flavipila (Bitter) M. Kessler \& Schmidt-Leb. & $3650-4100$ & HV, LI \\
Polylepis incana Humboldt, Bonpland \& Kunth & $3000-4200$ & AN, AP, AY, CU, HU, JU, LI, PA, PU \\
Polylepis incarum (Bitter) M. Kessler \& Schmidt-Leb. & $3100-4200$ & CU, PU \\
Polylepis lanata (Kuntze) M. Kessler \& Schmidt-Leb. & $2900-4100$ & AP, AY, CU \\
Polylepis microphylla (Wedd.) Bitter & $3200-4000$ & AR, CU, LI \\
Polylepis multijuga Pilger & $2200-3600$ & AM, CA, LA \\
Polylepis pauta Hieron. & $1800-4000$ & AY, CU, JU, SM \\
Polylepis pepei B.B. Simpson & $3900-4500$ & AN, CU, PU, SM \\
Polylepis racemosa Ruiz \& Pav. & $2900-4000$ & AN, AP, AY, CA, CU, HU, JU, LI, LL, PA \\
Polylepis reticulata Hieron. & $3350-4450$ & AN, JU, LI, LL \\
Polylepis rugulosa Bitter & $3000-4600$ & AR, MO, TA \\
Polylepis sericea Wedd. & $2000-4100$ & AN, CU, JU, LL \\
Polylepis subsericans J.F. Macbride & $2900-5100$ & AP, AY, CU \\
Polylepis subtusalbida (Bitter) M. Kessler \& Schmidt-Leb. & $3000-4500$ & MO, TA \\
Polylepis tarapacana Philippi & $4200-4800$ & TA \\
Polylepis tomentella Weddell & $3500-4500$ & AP, AR, AY \\
Polylepis triacontandra Bitter & $3500-3900$ & PU \\
Polylepis weberbaueri Pilger & $2500-4200$ & AN, CA, LA, LI, PI \\
\hline
\end{tabular}

1986, Kessler 1995, Romoleroux 1996), que podría conducir a procesos de especiación (Schmidt-Lebuhn et al. 2006). Incluso se descubrió casos de hibridización entre Polylepis y el género Acaena (Roulet 1981, Romoleroux 1996, Kerr 2003). En las poblaciones ecuatorianas, basado principalmente en el estudio de las relaciones morfológicas, se han observado altos niveles de hibridización (Segovia-Salcedo 2000), mientras que en el Perú no es muy frecuente observar poblaciones hibridas. Esto nos lleva a recomendar que los programas de reforestación, principalmente en las áreas protegidas por el estado peruano, eviten utilizar otras especies que no sean del mismo lugar para evitar la formación de híbridos; debido a que la hibridización reduce la tasa de reproducción, asimismo la habilidad de competir y detectar patógenos o depredadores (Levín et al. 1996, Rieseberg 1991, Soltis \& Gitzendanner 1998, Segovia-Salcedo 2000), pudiendo causar el decrecimiento pasivo de las poblaciones, como ocurrió con dos población de P. serícea en Ecuador (Segovia 2000).

Los resultados de este trabajo indican que el Perú es el país con mayor riqueza y endemismo específico para el género Polylepis. Lo que podría atribuirse a la presencia de poliplodía dentro del género, proceso muy importante dentro de la diversificación de las especies (Quija-Lamina et al. 2010), sumado a la gran diversidad ecológica y climática que posee el territorio peruano, que está determinado principalmente por dos factores, la cordillera de los Andes y la corriente peruana, esta última modifica notablemente las condiciones térmicas y la precipitación pluvial en la costa y la vertiente occidental y la primera crea una gran diversidad de zonas ecológicas entre las estribaciones de los Andes: valles profundos, altiplanicies y grandes cumbres (Milla Batres 2002). La cordillera andina en el Perú está conformada por la cordillera occidental (muy árida) y oriental (muy húmeda) que corren casi paralelas y están divididas a su vez en valles longitudinales y transversales profundamente cortados de esta manera surgen los valles interandinos. Los bosques de Polylepis en el Perú están establecidos principalmente en la parte alta, tanto de la cordillera occidental, oriental y los valles interandinos de todo el territorio peruano.
Probablemente el género se origino en los Andes del norte del Perú, ya que esta región alberga dos de las especies consideradas más primitivas ( $P$. multijuga, $P$. pauta y $P$. lanuginosa), por compartir varios caracteres morfológicos con Acaena, como la corteza bastante delgada, hojas grandes con numerosos folíolos, y una gran inflorescencia con muchas (hasta 80) flores (Kessler 1995, Fjeldsa \& Kessler 1996); una de ellas restringida a este sector de los Andes (Polylepis multijuga); una vez desarrollada como árbol altoandino, pudo colonizar áreas de hábitats, que hasta ese momento se encontraban casi completamente desprovistos de árboles en el Pleistoceno; de donde se disperso a lo largo de los Andes hacia el norte y sur (Van Der Gammen \& Cleef 1983, Troll 1959, 1968,).

Los departamentos más diversos para el género Polylepis son Cusco y Ayacucho, estos se encuentran en la región considerada en el presente trabajo, como el centro de diversificación del género Polylepis. Es preciso indicar que la distribución departamental en el Perú, aunque útil, muestra limitantes desde la perspectiva biológica, ya que la división política no refleja una diferencia ambiental o histórica. Por tal motivo es necesario analizar la distribución natural (no reforestados) de las especies de Polylepis, basado en factores climáticos y edáficos, la que permitirá conocer más sobre la biogeografía del género.

Los datos de distribución altitudinal, muestran que el intervalo en el que se desarrolla las especies de Polylepis en el Perú es muy amplio, ya que abarca desde los 1800 hasta los 5100 m de altitud; sin embargo, las especies (18) se presentan más frecuentemente entre los 3000 y $4000 \mathrm{~m}$ de altitud; lo mismo ocurre con las especies de Polylepis en Bolivia, el 100\% (13) de las especies son frecuentes entre los 3000 y $4000 \mathrm{~m}$, el registro a mayor altitud en comparación con el resto de los países donde ocurre el género Polylepis, se encuentra en Bolivia, en la localidad de Sajama sobre los 5200 m (P. tarapacana). Podemos también concluir que Polylepis tiene una amplia tolerancia ecológica, por distribuirse desde áreas muy húmedas (yungas), hasta las áreas xerofíticas (puna seca). 


\section{Agradecimientos}

Nuestro agradecimiento a los curadores de los: Herbario de Missouri (MO), Field Museum of Natural History (F), Herbarium U.S. National Arboretum (Washington D.C. NA), Herbario de San Marcos (USM), Herbario de San Agustín de Arequipa (HUSA), Herbario de César Vargas de Cusco (CUZ) y Herbario de la Universidad Nacional de Cajamarca (CPUN). Asimismo, al Proyecto Queñual, al Consejo Nacional de Ciencia y Tecnología (CONCYTEC) por financiar el estudio de los Polylepis; así como a la Universidad Nacional Mayor de San Marcos, por el financiamiento a los proyectos liderados por el segundo autor. A Mónica Arakaki y Rosa Vento por la revisión del abstract.

\section{Literatura Citada}

Bitter G. 1911. Revision del gattung Polylepis. Botanische Jahrbücher Für Systematik, Pflanzengeschichte und Pflanzengeographie. 45:564-656.

Brako L. \& J.L. Zarucchi. 1993. Catálogo de las Angiospermas y Gimnospermas del Perú. Monographs in Systematic Botany from the Missouri Botanical Garden. Volumen 45. $1286 \mathrm{pp}$.

Cabido M. \& A. Acosta. 1985. Estudio Fitosociológico en bosque de Polylepis australis Bitter: ("Tabaquillo") en las sierras de Córdoba. Argentina. Documents phytosociologiques. N.S. Vol. IX: 365-400.

Fjeldsa J. \& M. Kessler. 1996. Conserving The Biolgical Diversity Of Polylepis Woodlands of the Highland of Perú and Bolivia. A Contribution to Sustainable Natural Resource Management in the Andes, NORDECO, Copenhagen, Dermark. 250 pp.

Galiano W. 1990. The flora of Yanacocha, a tropical high-Andean forest in Souther Perú. University of Missouri, St Louis. Department of Biology, St. Louis, EEUU. Tesis de Master. $243 \mathrm{pp}$.

Herrera F.L. 1943. Sinopsis de las especies del género Polylepis (La Qqueuña). Boletín del Museo de Historia Natural "Javier Prado". Año VII. (26 y 27):219-228.

Kerr M.S. 2003. A Phylogenetic and biogeographic analysis of Sanguisorbeae (Rosaceae) with emphasis on the Pleistocene radiation of the High Andean genus Polylepis. PhD thesis, University of Maryland. $191 \mathrm{pp}$.

Kessler M. 1995. Polylepis Walder Boliviens: Taxa Okologie, Verbreitung and Geschichte. Dissertationes Botanicae 246. J. Cramer. Berlin, Stuttgart. 303 pp.

Kessle M. \& A.N. Schmidt-Lebuhn. 2006. Taxonomical and distributional notes on Polylepis (Rosaceae). Org. Divers. Evol. 6, Electr. Suppl. 1:1-10.

Koepcke M. 1961. Birds of the western slope of the Andes of Peru. American Museum Novitates. 2028: 1-31.

Levín D.A.; J. Francisco-Ortega \& R.K. Jansen. 1996. Hybridization and the extinction of rare plant species. Conservation Biology 10(1): 10-16.

Macbride J.F. 1938. Flora of Perú. Volumen XIII, parte II. Botanical Series. Field Museum Natural History: 1036-1119.

Mendoza W. 2005. Especie nueva de Polylepis (Rosaceae) de la cordillera Vilcabamba (Cusco, Perú). Rev. Peru. Biol. 12(1): 103-106.

Mendoza W. 2010. Taxonomía y distribución de las especies peruanas de Polylepis Ruiz \& Pav. (Rosaceae, Magnoliopsida). Tesis de Magister. UNMSM. Lima-Perú. 125 pp.
Mendoza W., A. Cano \& R. Vento. 2010. Bosques de Polylepis de la Reserva Nacional de Salinas y Aguada Blanca, Arequipa y Moquegua, Perú. Pp. 167-173. En H. Zeballos, J. A. Ochoa \& E. Lopez (Eds): Biodiversidad de la Reserva Nacional de Salinas y Aguada Blanca. DESCO, PROFONANPE, SERNANP. 313 P.

Milla Batres C. (ed.) 2002. Enciclopedia temática del Perú. La Flora Peruana. Tomo II. Lima: Editorial Milla Batres, (Impreso en Colombia) $220 \mathrm{pp}$.

Pérez de Paz J. 2004. Rosaceae-Sanguisorbae de Macaronesia: género Marcetella, Bencomia y Dendriopoterium. Palinología, Biogeografía, Sistema Sexual y Filogenia. Bot. Macaronesica 25: 95-126.

Quija-Lamina P., M.C. Segovia-Salcedo; M. Jadán \& K. Proaño. 2010. Estandarización de la metodología para el conteo cromosómico en especies del género Polylepis en el Ecuador. Revista Ecuatoriana de de Medicina y Ciencias Biológicas. Vol. XXXI Número 1 y 2: 33-49.

Riesenberg L.H. 1991. Hybridization in rare plants: insight from case studies in Cercocarpus and Helianthus. Pp. 171-181 en Falk, D.A \& K.E. Holsinger (eds.) Genetics and Conservations of Rare Plants, Oxford University Press, New York $283 \mathrm{pp}$.

Romoleroux K. 1996. Rosaceae. Pp 71-89 in Harling G. \& L. Anderson (eds.) Flora of Ecuador 56. Göteborg University. $151 \mathrm{pp}$.

Romoleroux, K. 1992. Rosaceae in the Páramo of Ecuador. In Balslev H. \& J.L. Luteyn (eds.) Páramo: An Andean Ecosystem under Human Influence: 85-94.

Roulet M. 1981. Contribution á l'etude du genre Acaena L. (Rosaceae) dans les Andes Argentines et Chiliennes. Bulletin de la Société Neucháteloise de Sciences Naturelles 104: 145-151.

Schmidt-Lebuhn A.N., M. Kessler \& M. Kumar. 2006. Promiscuity in the Andes: species relationships in Polylepis (Rosaceae, Sanguisorbeae) based on AFLP and morphology. Systematic Botany, 31(3): pp. 547-559.

Segovia-Salcedo M.C. 2000. Estudios fenéticos de Polylepis Ruiz \& Pavón en tres áreas de diversificación en Ecuador. En Freire A. \& D.A. Nelly (eds.). Memorias del Tercer Congreso Ecuatoriano de Botánica: 40-66.

Servat P.G., W. Mendoza \& J.A. Ochoa. 2002. Flora y Fauna de cuatro bosque de Polylepis (Rosaceae) en la Cordillera del Vilcanota (Cusco, Perú). Ecología Aplicada, 1(1): 25-35.

Simpson B.B. 1979. A revision of the genus Polylepis (Rosaceae: Sanguisorbae). Smithsonian Contributions to Botany. 43:1-61.

Simpson B.B. 1986. Speciation and specialization of Polylepis in the Andes. Pp.304-315 en Vuilleumier, F. \& M. Monasterio (eds.) High Tropical Biogeography, Oxford University Press, Oxford $671 \mathrm{pp}$.

Soltis S.P. \& M.A. Gitzendanner. 1998. Molecular Systematics and the conservation of rare species. Conservation Biology 13 (4): 471-483.

Troll C. 1959. Die tropishe Gebirge. Bonner geographische Abhandlungen. 25: 1-19.

Troll C. 1968. The Cordilleras of the Tropical Americas. Coll. Geogr. 9.

Van Der Hamment T. \& A.M. Cleef. 1983. Datos para la historia de la flora andina. Revista Chilena de Historia Natural. 56: $97-107$. 